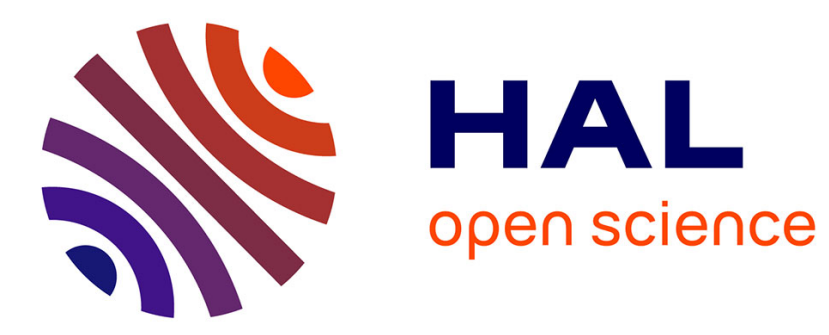

\title{
Non-parametric identification of the non-homogeneous stress in high strain-rate uni-axial experiments
}

\author{
Souheila Aloui, Ramzi Othman, Arnaud Poitou, Pierrick Guégan, Sami
}

El-Borgi

\section{- To cite this version:}

Souheila Aloui, Ramzi Othman, Arnaud Poitou, Pierrick Guégan, Sami El-Borgi. Non-parametric identification of the non-homogeneous stress in high strain-rate uni-axial experiments. Mechanics Research Communications, 2008, 35 (6), pp.392-397. 10.1016/j.mechrescom.2008.04.005 . hal-01007109

\section{HAL Id: hal-01007109 \\ https://hal.science/hal-01007109}

Submitted on 18 Jan 2017

HAL is a multi-disciplinary open access archive for the deposit and dissemination of scientific research documents, whether they are published or not. The documents may come from teaching and research institutions in France or abroad, or from public or private research centers.
L'archive ouverte pluridisciplinaire HAL, est destinée au dépôt et à la diffusion de documents scientifiques de niveau recherche, publiés ou non, émanant des établissements d'enseignement et de recherche français ou étrangers, des laboratoires publics ou privés. 


\title{
Non-parametric identification of the non-homogeneous stress in high strain-rate uni-axial experiments
}

\author{
Souheila Aloui ${ }^{\mathrm{a}}$, Ramzi Othman ${ }^{\mathrm{a}}$, Arnaud Poitou ${ }^{\mathrm{a}}$, Pierrick Guégan ${ }^{\mathrm{a}}$, Sami El-Borgi ${ }^{\mathrm{b}}$ \\ a Institut de Recherche en Génie Civil et Mécanique, Ecole Centrale de Nantes, 1 Rue de la Noë BP 92101, 44321 Nantes cedex 3, France \\ ${ }^{\mathrm{b}}$ Applied Mechanics and Systems Research Laboratory, Tunisia Polytechnic School, BP 743, 2048 La Marsa, Tunisia
}

\begin{abstract}
Stress and strain homogeneity, in tested samples, is a crucial assumption during a dynamic test. Whenever this assumption is true, the conventional recovery of the mean strain and stress is valid. However, when the stress and strain fields in the sample are not homogeneous, more sophisticated treatment must be considered. Inverse problem techniques are then proposed. Nevertheless, they may yield a non-physical result. In this paper, a non-parametric solution to the problem of non-homogeneity in dynamic tests is presented. The stress field is deduced from the displacement field measured via a Digital Speckle Photography (DSP) technique and a force boundary condition.
\end{abstract}

\author{
Keywords: \\ Hopkinson bar \\ Dynamic equilibrium \\ High strain-rate \\ Soft materials \\ Digital Speckle Photography
}

\section{Introduction}

Several techniques have been developed for measuring the stress-strain relationship at a wide range of strain-rates (Field et al., 2004). With all of these techniques, displacement and force measurements are recorded at a part of the specimen boundary. The stress and the strain within the specimen are then obtained, from these boundary conditions, assuming that they are homogeneous during the test. Indeed, only the mean values of stress and strain are approximated in this way. However, the assumption of homogeneity is not valid when testing soft materials and for high rates of loading (Song and Chen, 2004; Pattofatto et al., 2006). In these cases, the conventional recovery of the mean strain, stress and strain-rate can lead to inaccuracies (Meng and Li, 2003). Hence, many authors (Rota, 1997; Kajberg and Lindvist, 2004; Kajberg and Wikman, 2007; Diot et al., 2007), proposed the use of inverse recovery techniques. However, these techniques have some drawbacks: (i) the form of the constitutive equation of the material has to be known beforehand, (ii) the result depends on the chosen constitutive equation and (iii) the recovered stresses and strains do not always have a physical sense.

Digital speckle measurements are a powerful technique for measuring the displacement field in a tested specimen. By use of high-speed video cameras, these techniques can be applied to dynamic tests (Kajberg and Lindvist, 2004; Guetari et al., 2006; Kajberg and Wikman, 2007). Then, the strain field is directly deduced from the measured displacement field without the assumption of homogeneity. In this paper, we intend to extend this technique to measure the stress field. It is valid even in the case of a non-homogeneous state of stress or strain. To the best of our knowledge, this has never been done before. 


\section{Theory}

Let $\Omega(t)$ be the domain occupied by the tested specimen during the test. Each point in $\Omega(t)$ is represented by its position $\underline{X}=X_{1} \underline{e_{1}}+X_{2} \underline{e_{2}}+X_{3} \underline{e_{3}}$ in the initial configuration $\Omega(0)=\Omega_{0}$. We limit our development to the case of uni-axial tests; mainly, tensile-compression tests (see Fig. 1). Therefore, we can assume that: (i) the stress field is uni-axial, (ii) the cross-sections remain plane during the test and (iii) the volumetric forces are insignificant. Consequently, the stress field is reduced to one component $\sigma_{11}$ which only depends on time and $X_{1}: \sigma_{11}\left(X_{1}, t\right)$. Following Meng and Li (2003), we define the stress homogeneity coefficient:

$$
\alpha_{\sigma_{11}}(t)=\frac{\int_{0}^{L}\left|\sigma_{11}\left(X_{1}, t\right)-\left\langle\sigma_{11}\right\rangle(t)\right|^{2} \mathrm{~d} X_{1}}{\int_{0}^{L}\left|\left\langle\sigma_{11}\right\rangle(t)\right|^{2} \mathrm{~d} X_{1}},
$$

where $\left\langle\sigma_{11}\right\rangle(t)=\int_{0}^{L} \sigma_{11}\left(X_{1}, t\right) \mathrm{d} X_{1} / L .\left\langle\sigma_{11}\right\rangle(t)$ is the mean value of $\sigma_{11}\left(X_{1}, t\right)$. In conventional dynamic tensile-compression tests, only this mean value is recovered. Actually, it is approximated by one or two boundary conditions. Indeed, one or two force measurements are recorded at $X_{1}=0$ and $X_{1}=L$, say $F_{0}(t)$ and $F_{L}(t)$. The mean value of the stress is approximated as follows:

$$
\left\langle\sigma_{11}\right\rangle(t) \approx \frac{F_{0}(t)}{A(t)}, \text { or } \quad\left\langle\sigma_{11}\right\rangle(t) \approx \frac{F_{0}(t)+F_{L}(t)}{2 A(t)},
$$

where $A(t)$ is a mean value of the $e_{1}$-normal cross-section areas. Usually, $A(t)$ is computed by assuming an iso-volume test. In the case of a stress field which does not fluctuate very much around the mean value, i.e., $\alpha_{\sigma_{11}} \ll 1$, Eq. (2) represent accurately the stress field. However, in very high strain-rate or soft material testing, $\alpha_{\sigma_{11}}$ may not be small throughout the test. In such a case, Eq. (2) are not sufficient.

In this work, we are interested in determining the variation of $\sigma_{11}$ along the $e_{1}$-direction, i.e., $\sigma_{11}\left(X_{1}, t\right)$. Let $S(x)=\left\{\underline{X} \in \Omega_{0}, \underline{X} . e_{1}=x\right\}, F_{1}\left(X_{1}, t\right)$ and $A\left(X_{1}, t\right)$ be the cross-section of normal $e_{1}$ at $X_{1}=x$, the variation of the normal force (longitudinal force) on $S\left(X_{1}\right)$ and the variation of the $S\left(X_{1}\right)$-area, respectively. The momentum equation written on the elementary volume between $X_{1}$ and $X_{1}+\mathrm{d} X_{1}$ (Fig. 1) yields

$$
\frac{\partial F_{1}\left(X_{1}, t\right)}{\partial X_{1}}=\rho_{0} A\left(X_{1}, 0\right) \frac{\partial^{2} u_{1}\left(X_{1}, t\right)}{\partial t^{2}}
$$

where $\rho_{0}$ is the initial density of the material and $u_{1}$ the displacement of $S\left(X_{1}\right)$ in the $\underline{e}_{1}$-direction (longitudinal displacement). Eq. (3) is equivalently rewritten

$$
F_{1}\left(X_{1}, t\right)=F_{1}(0, t)+\int_{\xi=0}^{\xi=X_{1}} \rho_{0} A(\xi, 0) \frac{\partial^{2} u_{1}(\xi, t)}{\partial t^{2}} \mathrm{~d} \xi .
$$

The nominal $\sigma_{11}^{n}\left(X_{1}, t\right)$ and the true $\sigma_{11}^{\mathrm{tr}}\left(X_{1}, t\right)$ stresses are then recovered as follows:

$$
\sigma_{11}^{n}\left(X_{1}, t\right)=\frac{F_{1}\left(X_{1}, t\right)}{A\left(X_{1}, 0\right)} \quad \text { and } \quad \sigma_{11}^{\mathrm{tr}}\left(X_{1}, t\right)=\frac{F_{1}\left(X_{1}, t\right)}{A\left(X_{1}, t\right)} .
$$

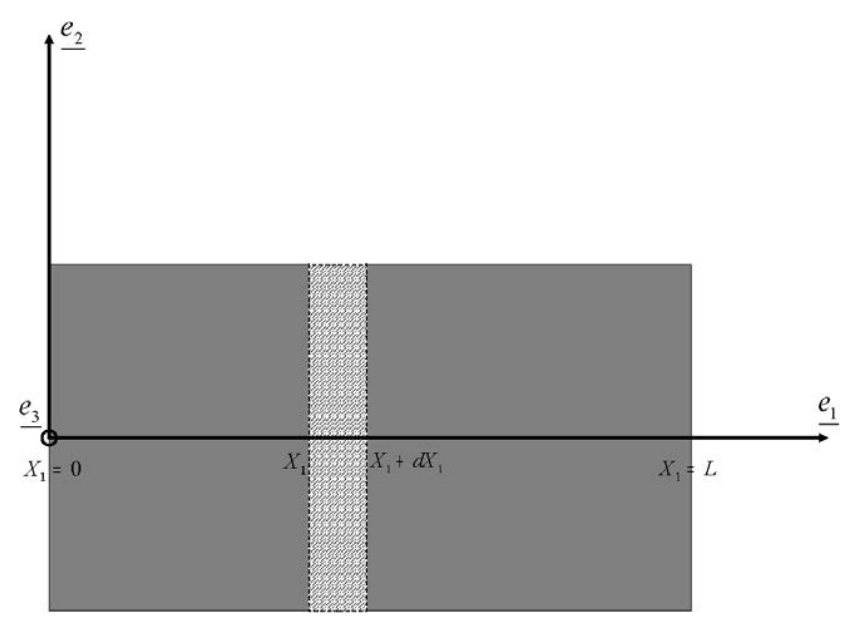

Fig. 1. Simplified scheme of a compression or tensile test specimen. 
Eqs. (4) and (5) mean that the longitudinal force, nominal stress and true stress can be determined at any cross-section $S\left(X_{1}\right)$ if $F_{1}(0, t), u_{1}\left(X_{1}, t\right)$ and $A\left(X_{1}, t\right)$ are known. $F_{1}(0, t)$ can be measured using a conventional force sensor whereas $u_{1}\left(X_{1}, t\right)$ and $A\left(X_{1}, t\right)$ can be determined using a DSP technique. Precisely, the variation of a cross-section area can be written as follows:

$$
A\left(X_{1}, t\right)=A\left(X_{1}, 0\right)\left(1+\epsilon_{22}^{n}\right)\left(1+\epsilon_{33}^{n}\right)
$$

where the superscript ' $n$ ' stands for the nominal values. In the case of an isotropic material, $\epsilon_{22}^{n}=\epsilon_{33}^{n}$. Hence, Eq. (6) is simply rewritten

$$
A\left(X_{1}, t\right)=A\left(X_{1}, 0\right)\left(1+\epsilon_{22}^{n}\right)^{2} .
$$

Consequently, only one camera is necessary when testing isotropic materials. Indeed, the cross-section area variation can be deduced from the in-plane displacements. However, a second camera has to be used in the case of orthotropic materials.

\section{Numerical validation}

In this section, we are interested in validating the method presented above on a numerical test. Indeed, a dynamic compressive test is simulated on a rubber-like prismatic specimen using the commercial code ABAQUS 6.2. The sample is $20.2 \mathrm{~mm}$ in length, $19.9 \mathrm{~mm}$ in height and $30 \mathrm{~mm}$ in width. For $t>0$, we impose two boundary conditions: $u_{1}(0, t)=0$ and $v_{1}(L, t)=0.2 \mathrm{~m} / \mathrm{s}$, where $v_{1}$ is the longitudinal particle velocity. Due to symmetry, only one-fourth of the geometry is modelled. In addition, we assume that the constitutive equation of the material follows the Ogden law (Ogden, 1972). For a third order law, the Ogden potential is written

$$
\left.\psi=\sum_{i=1}^{3} \frac{\mu_{i}}{\alpha_{i}}-3+\sum_{j=1}^{3} \lambda_{j}^{\alpha_{i}}\right)
$$

where $\left(\lambda_{i}\right)_{i=1,3}$ are the principle widening of Cauchy-Green tensor, $\left(\mu_{i}\right)_{i=1,3}$ and $\left(\alpha_{i}\right)_{i=1,3}$ are material parameters. The chosen values of these parameters are given in Table 1 . The simulation was carried out on $10 \mathrm{~ms}$ with an automatically generated step time. The specimen was meshed using an 8-node linear brick. The simulations outputs was generated every $\Delta t=250 \mu$ s. This choice is explained by the fact that maximum frequency of our video camera is 4000 pictures per second. Using the numerical model, one can access to the $u_{1}^{\mathrm{ab}}\left(X_{1}, t\right), A^{\mathrm{ab}}\left(X_{1}, t\right), F_{1}^{\mathrm{ab}}(0, t)$ and $\sigma_{11}^{\mathrm{tr} a \mathrm{~b}}\left(X_{1}, t\right)$, where the superscript 'ab' means ABAQUS values. A white noise is added to numerical signals to simulate experimental noise. The variance of the numerical noise is taken $1 \%$ of the maximum value of each signal.

It is worth noticing that the Discrete Fourier Transforms with low-pass filter are used to compute the acceleration $\frac{\partial^{2} u_{1}\left(X_{1}, t\right)}{\partial t^{2}}$ from the longitudinal displacement. The cutting frequency is taken half the maximum frequency. Using Eqs. (4) and (5) we can determine the true stress given by the new method. It will be denoted $\sigma_{11}^{\text {tr, } h h}$. The left equation in Eq. (2) is also used to recover the true stress as approximated by the conventional method. It will be denoted $\sigma_{11}^{\text {tr, } h}$. The errors of the new method and the conventional one are defined by

$$
\begin{aligned}
& \zeta_{n h}\left(X_{1}, t\right)=\frac{\left|\sigma_{11}^{\mathrm{tr}, n h}\left(X_{1}, t\right)-\sigma_{11}^{\mathrm{tr}, a b}\left(X_{1}, t\right)\right|^{2}}{\left|\sigma_{11}^{\mathrm{tr}, a b}\left(X_{1}, t\right)\right|^{2}}, \text { and } \\
& \zeta_{h}\left(X_{1}, t\right)=\frac{\left|\sigma_{11}^{\mathrm{tr}, h}\left(X_{1}, t\right)-\sigma_{11}^{\mathrm{tr}, \mathrm{ab}}\left(X_{1}, t\right)\right|^{2}}{\left|\sigma_{11}^{\mathrm{tr}, \mathrm{ab}}\left(X_{1}, t\right)\right|^{2}},
\end{aligned}
$$

respectively. In Fig. $2, \zeta_{n h}$ is compared to $\zeta_{h}$. As expected, the error of the conventional method is large in the beginning of the test when the stress field is non-homogeneous and slow down thereafter. The error of the new technique $\zeta_{n h}$ is lower than that of the conventional technique $\zeta_{h}$. As the proposed technique takes into account the stress non-homogeneity, $\zeta_{n h}$ does not vary much during the test and it is of a few percent.

\section{Experimental results and discussion}

After checking the validity and the accuracy of the proposed technique, we will apply this non-parametric identification technique to an MTS machine test on a synthetic rubber (Cribex 80) specimen. A simplified scheme of the experimental setup is given in Fig. 3. The dimension of the sample and the density are the same as those of the numerical sample. The load is applied by the lower cross beam which moves with a velocity equal to $0.2 \mathrm{~m} / \mathrm{s}$. On the fixed jack, a piezoelectric force sensor records $F_{1}(0, t)$. On the other hand, the displacement field is measured by a high speed video camera (PHOTRON Ultima APX)

Table 1

Rubber-like parameters for Ogden law

\begin{tabular}{llllll}
\hline Parameter & $\mu_{1}$ & $\mu_{2}$ & $\mu_{3}$ & $\alpha_{1}$ & $\alpha_{2}$ \\
\hline Value & $3.25 \times 10^{7}$ & $-2.98 \times 10^{7}$ & 360,000 & 2.01 & 1.82 \\
\hline
\end{tabular}




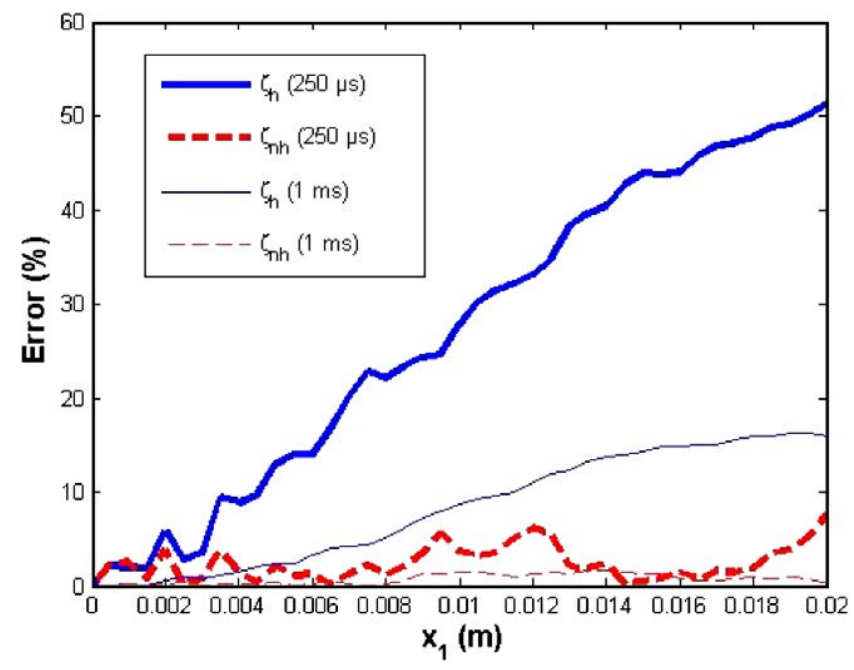

Fig. 2. Error of the new method $\left(\zeta_{n h}\right)$ and the conventional method $\left(\zeta_{h}\right)$.

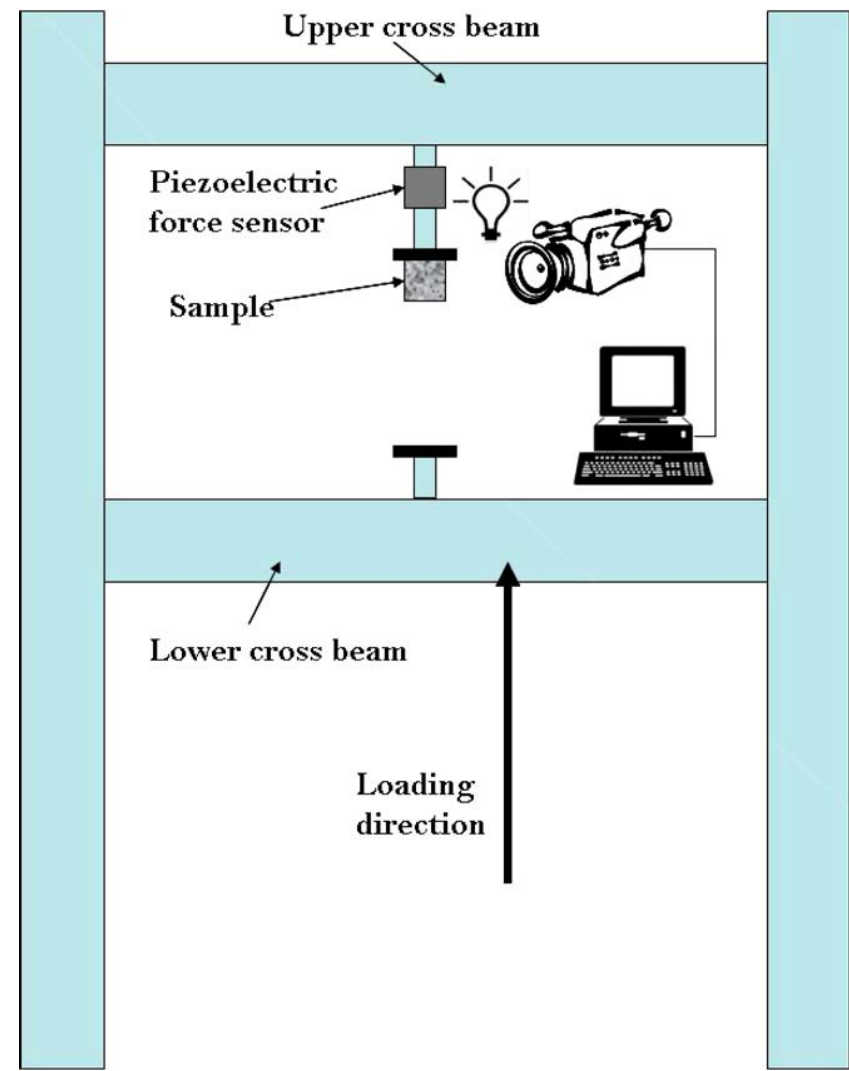

Fig. 3. Simplified scheme of the experimental set-up.

and a DSP (Digital Speckle Photographs) technique. Photographs are taken at a rate of 4000 pictures a second. The specimen photography taken at a time $t$ is compared to the a reference photography (in our study the reference photography is taken at $t=0$ ). Then a digital cross-correlation algorithm is applied to the two selected regions (Field et al., 2004) and calculates the in-plane displacement field. The nominal and true (logarithmic) in-plane strain can also be deduced from the displacement field using the conventional equations of continuum mechanics. In our study, the recorded photographs are analysed by the 


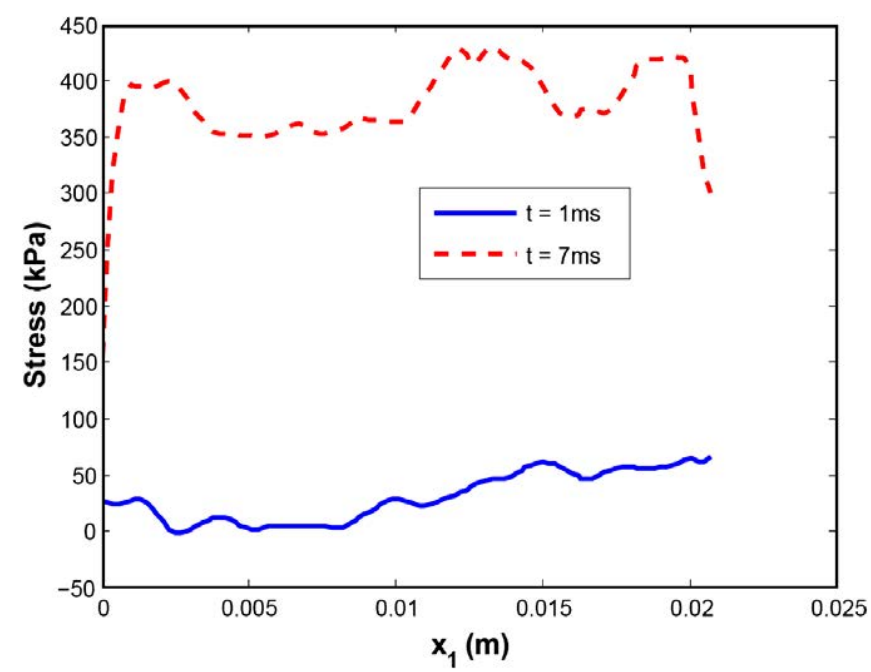

Fig. 4. Stress variation in the specimen.

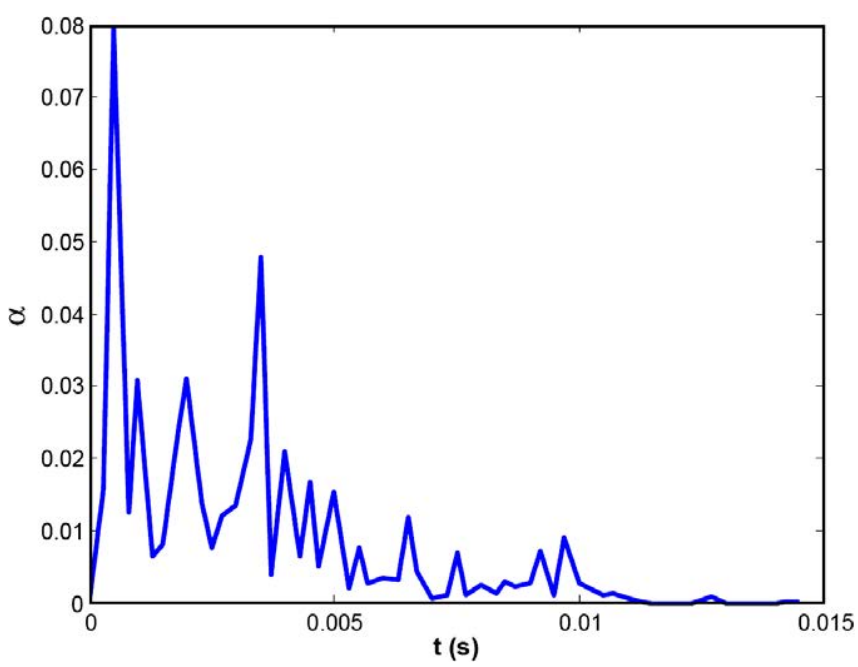

Fig. 5. Coefficient of the stress homogeneity: $\alpha=\alpha_{\sigma_{11}}$.

ICASOFT software, developed at the INSA Lyon, yields the displacement $u_{1}\left(X_{1}, t\right)$ and the strain $\epsilon_{22}\left(X_{1}, t\right)$. Eq. (7) is used to infer $A\left(X_{1}, t\right)$. Then, $\sigma_{11}^{\text {tr, } h h}\left(X_{1}, t\right)$ is recovered by applying Eqs. (4) and (5).

In Fig. 4, we show the recovered stress field after $1 \mathrm{~ms}$ and $7 \mathrm{~ms}$. Additionally, we can also measure the coefficient of stress homogeneity $\alpha_{\sigma_{11}}(t)$ (see Fig. 5). As expected, the stress field is non-homogeneous in the beginning of the test. By the end of the test $\alpha_{\sigma_{11}}(t)$ vanishes. The oscillation of the homogeneity coefficient would be attributed to wave propagation in the sample and/or the method inaccuracies. The numerical derivation of the displacement field and the low frequency of the video camera could induce these problems. However, the method still give better results than the conventional technique. Using signal processing techniques and video camera with higher frequency should improve the accuracy of the method. It is worth noticing that the coefficient of stress homogeneity is only determined numerically in Meng and Li (2003). It is now possible to measure this coefficient.

\section{Conclusion}

In this paper, a non-parametric method is presented to measure the stress field in dynamic tests even in the case of a nonequilibrium state. This is an alternative solution to the inverse recovery techniques. This method is tested numerically. It is shown that it gives good precision. Its accuracy is much better than that of the conventional recovery method. Using signal processing techniques and video camera with higher frequency should yield to better accuracy. This method gives access to 
the homogeneity coefficients in the sample during a dynamic test, which, by the past, was only possible numerically. The authors are convinced that the proposed solution would open another application field to DSP techniques.

\section{Acknowledgements}

The Region Pays de la Loire (France) is highly acknowledged for the financial support to the first author. Furthermore, the authors would like to thank B. Lundberg (Uppsala University, Sweden) and E. Verron (Ecole Centrale de Nantes) for helpful discussions and F. Pasco (Ecole Centrale de Nantes) for his help in the experimental work.

\section{References}

Diot, S., Guines, D., Gavrus, A., Ragneau, E., 2007. Two-step procedure for identification of metal behavior from dynamic compression tests. Int. J. Impact Eng. 34, 1163-1184.

Field, J.E., Walley, S.M., Proud, W.G., Goldrein, Siviour, C.R., 2004. Review of experimental techniques for high rate deformation and shock studies. Int. J. Impact Eng. 30, 725-755.

Guetari, Y., Guegan, P., Poitou, A., 2006. An experimental investigation of orthogonal cutting advanced measurement techniques and machinability aspects. Int. J. Forming Processes 9, 473-489.

Kajberg, J., Lindvist, G., 2004. Characterisation of materials subjected to large strain rates by inverse modelling based on in-plane displacement fields. Int. J. Solids Struct. 41, 3439-3459.

Kajberg, J., Wikman, B., 2007. Viscoplastic parameter estimation by high strain-rate experiments and inverse modelling - Speckle measurements and highspeed photography. Int. J. Solids Struct. 44, 145-164.

Meng, H., Li, Q.M., 2003. Correlation between the accuracy of a SHPB test and the stress uniformity based on numerical experiments. Int. J. Impact Eng. 28, $537-555$.

Ogden, R.W., 1972. Large deformation isotropic elasticity: on the correlation of the theory and experiment for compressible rubberlike solids. Proc. Roy. Soc. Lond. A 328, 567-583.

Pattofatto, S., Poitou, A., Tsitsiris, H., Zhao, H., 2006. A new testing method to investigate the compacting behaviour of fresh concretes under impact loading. Exp. Mech. 46, 377-386.

Rota, L., 1997. Application des méthodes inverses au dépouillement de l'essai aux barres de Hopkinson. Ph.D. Thesis, Ecole Polytechnique, France.

Song, B., Chen, W., 2004. Dynamic stress equilibration in split Hopkinson pressure bar tests on soft materials. Exp. Mech. 44, 300-312. 